\title{
Cognitive and psychosocial deficits in agenesis of the corpus callosum with normal intelligence
}

\author{
Warren S. Brown \\ Fuller Graduate School of Psychology, Pasadena, USA \\ and the University of California, Los Angeles, USA \\ Lynn K. Paul \\ Fuller Graduate School of Psychology, Pasadena, USA
}

\begin{abstract}
Introduction. Cognitive disabilities in agenesis of the corpus callosum (ACC) have been described in scattered reports, but few complete case descriptions are available. Consequent psychosocial disabilities in ACC have received little attention. We expected that ACC would be manifest in deficits specific to complex reasoning, concept formation, and problem solving. We also expected that these cognitive problems would be particularly evident in difficulties interpreting and understanding social situations, and in abnormal self-perception. Methods. Cognitive and psychosocial deficits were studied in two adolescents with ACC and normal IQ using a battery of cognitive and psychological tests. Results. Test results indicated poor interhemispheric integration of complex material. Performance on tests of reasoning, and concept formation were clearly below expectations based on IQ. Significantly poor performance was also found on tests of social insight, proverb interpretation, social logic, self-perception, and interpretation of ambiguous stimuli. Gross behavioural disorder or psychopathology were not found. Conclusions. ACC results in disabilities in social cognition that appear to be secondary to deficits in complex cognitive operations such as reasoning, concept formation, and problem solving. It is suggested that these cognitive deficits may be related to diminished interhemispheric transfer of complex information.
\end{abstract}

Correspondence should be addressed to Dr Warren S. Brown, The Travis Institute, 180 N. Oakland Avenue, Pasadena, CA 91101, USA.

This research was supported in part by NICHD Grant No. 1 R15 HD33118-01A1. The authors would especially like to thank Professor Malcolm Jeeves and Dr Joseph Bogen for very helpful comments on this manuscript, and Dr Connie Dunn and Beatrix Schieffer for participation in some of the patient testing. 


\section{INTRODUCTION}

The specific contribution of the corpus callosum to mental processes (both cognitive and psychosocial) is not yet entirely understood. Although the study of individuals with agenesis of the corpus callosum (ACC) has contributed important information toward answering this question, most of the individuals studied thus far have been well below the normal range of general intelligence. Where the intelligence of ACC subjects has been normal, the majority of studies have typically assessed narrow domains of cognitive ability or specific forms of interhemispheric transfer (see reviews by Chiarello, 1980; Sauerwein, Nolin, \& Lassonde, 1994). There is a dearth of literature on the consequences of ACC for social and psychological functioning.

This paper presents case studies of two young men with ACC and normal intelligence. The cognitive and psychosocial functioning of these two individuals is examined in a wide range of cognitive and psychosocial functions in an effort to develop a better understanding of the cognitive deficits that are specific to callosal agenesis, and the consequent difficulties in social understanding. According to reports from their families, the social functioning of both of these individuals has been more remarkable than their mild cognitive disabilities. Both sets of parents were concerned about their son's poor social insight and peer relationships. On an informal instrument, the parents rated their sons as missing the point of subtle stories and jokes, having difficulty problem solving in novel situations, and displaying poor social judgement.

The central hypothesis of this research was that the most important specific neuropsychological consequence of $\mathrm{ACC}$ is a reduction in the interconnectivity of brain systems and a consequent reduction in the richness of cortical networks available for higher cognitive information processing. The consequences of this reduction in interconnectivity are typically not apparent in tasks demanding rote and overlearned cognitive skills; thus, measured IQ may be normal. However, more complex forms of reasoning, concept formation, and problem solving are maximally demanding of access to multiple processing systems, and particularly of bihemispheric processing interactions. Thus, deficits will be found in more complex and novel cognitive processes. Finally, it is our hypothesis that accurate self-perceptions and age-appropriate social perceptions and interpretations demand ongoing complex cognitive processing such as reasoning, concept formation, and problem solving (Paul \& Brown, 1996).

A version of this theory was previously advanced by Bogen and Bogen (1969), in a paper entitled "The Corpus Callosum and Creativity". These authors suggested that the reduction in bihemispheric interconnectivity has an effect on problem solving. Bogen and Bogen argued: (1) that the hemispheres are capable of independent and somewhat different forms of information processing; (2) that having two independent processors is advantageous in problem solving; (3) to take advantage of the greater problem-solving power of 
two processors there must be a corpus callosum available for hemispheric interaction; and (4) that creativity in problem solving springs from the interaction of the two hemispheric processors. A subtheme of their argument is that bihemispheric processing via a corpus callosum is only necessary or particularly advantageous in more complex reasoning, concept formation, and problem solving. Thus, restriction of interhemispheric interactions to the noncallosal commissures in callosal agenesis would be expected to diminish the capacity for complex cognitive operations, imagination, and consequent creativity. We would enhance the theory of Bogen and Bogen by suggesting that bihemispheric processing and callosal interactions are particularly important for psychological and social information processing as this form of information is particularly complex, subtle, relatively novel, and consistently demanding.

\section{Interhemispheric transfer in callosal agenesis}

Individuals with ACC show normal or near normal interhemispheric transfer in most tasks (most likely due to the presence of the anterior commissure in the majority of cases). However, acallosals have greater difficulty transferring or accurately comparing complex forms of perceptual information provided to each hemisphere (Chiarello, 1980). We have recently demonstrated that although extracallosal paths (most likely the anterior commissure, typically present in ACC) are capable of subserving bilateral comparisons of single letters in acallosals, the absence of the corpus callosum results in clear deficiencies in bilateral comparisons of more complex visual patterns (Brown et al., 1999). Similar results were reported by Gott and Saul (1978).

Deficits in acallosal patients in intermanual transfer of the spatial information of a tactile maze or formboard (the Tactual Performance Test, or TPT) have been reported (Ferriss \& Dorsen, 1975; Fischer, Ryan, \& Dobyns, 1992; Gott \& Saul, 1978; Jeeves, 1979; Russell \& Reitan, 1955; Sauerwein et al., 1994; Solursh et al., 1965). Particularly revealing regarding the importance of informational complexity in interhemispheric transfer deficits in acallosals is a series of reports of dominant versus nondominant hand performance on the TPT. Sauerwein et al. (1981) reported unimpaired nondominant hand TPT performance (normal transfer) in two acallosal subjects using a simplified (6 block) TPT formboard. In a later study of another two acallosal patients with normal intelligence, Fischer et al. (1992) found mildly impaired nondominant hand TPT performance using the intermediate ( 8 block) formboard. Finally, when retested on the adult (10 block) formboard, the nondominant hand performance of Sauerwein's two acallosal subjects was clearly abnormal (Sauerwein et al., 1994). Thus, interhemispheric transfer deficits on the TPT (dominant to nondominant hand) appear in acallosals only when there is a demand for the transfer of more complex tactile-spatial information. Jeeves (1979, p.468) concluded, "Alternative pathways ... can compensate for the 
absence of the corpus callosum either when only easy tasks are presented or when there is no time constraint on performance. Such alternative pathways may not, however, be able to handle information transmission called for by complex tasks or when there is a speed stress on performance.'

\section{Neurocognitive disabilities in ACC}

Reduced efficiency of interhemispheric integration of more complex information appears to have an impact on some aspects of intelligence and higher cognitive activity. In a review of the literature regarding general intelligence scores among acallosal subjects, Sauerwein et al. (1994) demonstrated that although acallosal subjects may not exhibit deficits on tests of general intelligence, more subtle cognitive deficits may be revealed through tests of specific subskills. It has been suggested that callosal agenesis influences the "fine-tuning" of certain cognitive and motor skills (Jeeves \& Milner, 1987; Sauerwein et al., 1994; Temple, Jeeves, \& Villarroya, 1990). For example, Temple and Ilsley (1994) report deficits in complex visuospatial construction (the Cornish Jigsaw) and complex visuospatial memory (delayed recall of the Rey Osterrieth figure).

With respect to reading, Temple and her colleagues (Jeeves \& Temple, 1987; Temple \& Ilsley, 1994; Temple, Jeeves, \& Vilarroya, 1989, 1990) found a specific impairment in phonological processing in ACC, particularly evident in an inability to formulate and recognise rhyme. Dennis (1981) and Sanders (1989) both found specific deficits in syntactic comprehension and metalinguistic understanding. Thus, although normally intelligent ACC children may have adequate lexical reading skills, they will likely exhibit language deficits under particular circumstances: (1) when faced with complex, novel words that require phonological decoding; (2) when faced with comprehension tasks that require an understanding of complex syntax; or (3) when language processing places a heavy demand on metalinguistic skills (e.g., interpretation of proverbs).

Little research has directly tested abstract reasoning, concept formation, and problem solving in ACC patients. Rourke (1989) describes an agenesis patient ("Mary") who had marked deficits on both the TPT and the Categories tests, indicating poor problem solving with respect to general IQ. Among two acallosal subjects with normal intelligence, Fischer et al. (1992) found inconsistencies in performance on abstract conceptualisation and problemsolving tasks such as the Category Test and the Wisconsin Card Sort.

\section{Psychosocial characteristics of ACC}

Although ACC individuals do not typically show gross forms of psychopathology, ACC children have been reported to exhibit mild forms of social and behaviour disturbance (Njiokiktjien \& Ramaekers, 1991; O'Brien, 1994; Rourke, 1989). However, the exact nature of these behavioural problems has 
not yet been clarified. O'Brien (1994) conducted a developmental survey of children with partial ACC, complete ACC, and Aicardi's syndrome (ACC with additional disabilities). Lack of emotional communication was a common trait among patients in all three groups. However, autistic-type deficits, such as social indifference, dysphoric mood, irritability, and fearfulness, were uncommon in partial and complete ACC. Jeeves and Temple (1987) reported that among ACC patients who had adequate expressive language skills, "meaningless" or "outof-place" conversation was particularly common. Rourke (1989) describes agenesis patient "Mary" as having deficiencies in age-appropriate adaptive skills.

Parents of ACC children commonly report that it is difficult to know at any moment what their child is feeling or experiencing emotionally (O'Brien, 1994). O'Brien posits that this characteristic may relate to the socioemotional deficit termed "alexithymia" (Nemia \& Sifneos, 1970; Sifneos, 1972). Alexithymia literally means "no words for feelings and moods." Common characteristics of alexithymia include lack of emotional expressiveness, poor fantasy life, concrete thought, feelings based on external events, and a tendency toward somatic complaints (Lesser, 1981; Sifneos, 1973). The relationship between hemispheric disconnection and alexithymia was supported in a study of 12 commissurotomised patients by Hoppe and Bogen (1977). Further, studies by TenHouten et al. $(1985,1987,1988)$ found high levels of psychosomatic personality structure and alexithymia in commissurotomised patients as indicated by their responses to the emotional content of films. These investigators concluded that lack of connection between the right hemisphere, where affective experiences are processed, and the left hemisphere, which controls verbalisation, results in an inability to express emotions (or alexithymia). With respect to ACC, Buchanan, Waterhouse, and West (1980) proposed a relationship between hemispheric disconnection and alexithymia based on a case study involving reduced verbal expression of emotion in an adult with ACC and normal intelligence. Similarities between commissurotomy and ACC patients with respect to alexithymia suggest that the corpus callosum is a critical contributor to the ability to experience and/or describe emotions.

\section{Summary}

In ACC individuals with normal general intelligence, the most important neuropsychological consequence of callosal agenesis seems to be deficiencies in abstract reasoning, concept formation, and problem solving. Although scattered results in the literature suggest problems in psychosocial functioning, this issue has not been explored in depth. We present two case studies of the cognitive and psychosocial abilities of individuals with ACC and normal intelligence in order to further evaluate the hypothesis that ACC is associated with specific problems in complex cognitive operations, and that these cognitive problems will manifest 
themselves in psychosocial deficits marked by a failure to adequately comprehend the complexity of psychological and social processes.

\section{METHODS}

\section{Subjects}

Two adolescent males diagnosed with ACC were tested. Both individuals signed and parents cosigned informed consent forms and were treated according to the American Psychological Association Ethical Principles (American Psychological Association, 1992).

Case 1. MM is a right-handed, Caucasian male who was 16-18 years old over the period of testing. MM was fortuitously diagnosed with ACC at age 8 when he participated in a study of regional brain metabolism (PET) in dyslexic boys (Patient 8 of Khanna et al., 1994). Due to abnormal appearance of the PET, he received an MRI that revealed ACC, as well as significant dilation of the posterior horns of the lateral ventricles and presence of an anterior commissure. Prior to the diagnosis of ACC, MM's only cognitive/psychological diagnosis was mild dyslexia, for which he had received various types of special education. Because his reading performance has normalised, it is clear that "dyslexia" was a misdiagnosis. However, he continued to struggle with math, science, and creative writing. MM has nevertheless been able to graduate from high school.

Case 2. JD is a left-handed, Caucasian-Hispanic American male who was 21-23 years old at the time of testing. At the age of $18 \mathrm{JD}$ received an MRI subsequent to a fainting spell, revealing ACC with presence of the anterior commissure. The MRI also revealed some heterotopic grey matter in both hemispheres and dilation of the right temporal horn of the lateral ventrical. He was previously diagnosed with Attention Deficit Disorder and was treated with Ritalin throughout elementary and high school. However, JD is clearly not hyperactive and no evidence of attention deficit was found in our administration of the Child Behavior Checklist to both JD and his mother. We presume that ADD was an incorrect diagnosis based on problems with complex memory wrongly attributed to a failure of attention. JD graduated from high school, but received various types of special education. He performs well on single word reading, but exhibits poor reading comprehension and has poor math skills.

\section{Tests administered}

General intelligence was tested with the Wechsler Intelligence Scale for Children-Revised (WISC-R; Wechsler, 1974). Although JD was beyond the appropriate age for this test when first seen in our laboratory, results of a WISC-R administered at the age of 16 were available. 
The Wide Range Achievement Test 3 (WRAT-3) is a test of academic skills in the areas of spelling, reading, and arithmetic (Jastak \& Wilkinson, 1984). It is applicable to individuals from early childhood through middle adult years.

Both the 6-block and the 10-block versions of the Tactile Performance Test (TPT) were given. This test requires that the subject place differently shaped blocks into their proper location on the test formboard while blindfolded, first with the dominant hand and then with the nondominant hand (Halstead, 1947). Successful performance of the nondominant hand relative to that of the dominant hand is believed to reflect sharing of spatial information between the hemispheres. Scores for each subject are compared to age-appropriate norms for the 10-block version, and to the most appropriate norms available for the 6block version (norms for 13-year-old males). Although these norms for the 6block version may overestimate the level of overall performance of our ACC subjects, because the corpus callosum has reached adult levels of development in normal 13-year-olds (Yakovlev \& Lecours, 1967), the relative dominant/ nondominant hand differences still provide a reasonably good estimate of interhemispheric transfer of spatial learning. The only adult norms available for the 6-block TPT involved 40- to 60-year-old subjects, not deemed appropriate for the two subjects reported herein.

The computerised administration of the Category Test (Halstead, 1947; Reitan \& Davison, 1974) was administered and demographically corrected normative data were used in interpreting the results (Heaton, Grant, \& Matthews, 1991). As normative data was not yet available below age 20, MM was compared with 20-year-old norms. The Category Test is a concept formation task in which the subject learns a theoretical principle by trial and error. Each of the seven subtests has a particular correct response principle that is maintained across trials within the subtest.

Raven's Colour Progressive Matrices is a measure of complex problem solving that uses primarily visual/spatial stimuli (Raven, 1965; Raven, Court, \& Raven, 1976). The Colour Version of the Raven's test is appropriate for children and adolescents aged 6 to 17, as well as for adults. Data for JD and MM were compared to adult norms (Yeudall et al., 1986).

The Letter and Number Series Tests from the Primary Mental Abilities Test (Thurstone \& Thurstone, 1949) were administered to assess complex problem solving and inductive reasoning ability (Schaie, 1983; Schaie, Willis, \& O'Hanlon, 1994). Each test involves the identification of the next letter or number in a rule-driven series. The subject must identify the rule underlying the series of letters or numbers, and select the next in that series.

The Minnesota Multiphasic Personality Inventory (MMPI-2; Hathaway \& McKinley, 1989) was administered using standardised administration and published norms for 18- to 29-year-olds. The MMPI-2 does not have norms for younger adolescents. However, conversion of MM's scores to the MMPI-1 equivalent (where age-appropriate norms are available) did not change the 
pattern of results, therefore MMPI-2 scores are reported. The MMPI-2 is a selfreport questionnaire that results in three factors for validity and 10 scales related to clinical symptoms associated with mood disturbance, thought disturbance, and physical concern (see the caption to Figure 2 for details).

The 12-item free-answer version and the 40-item best-answer version of the Proverbs Test (Gorham, 1956) were used to assess abstract verbal comprehension and reasoning. On the best-answer form, correct (abstract) responses were summed to attain an abstraction score. Normative data are designated by grade level from grade 5 to college juniors and seniors (Gorham, 1956). Because JD did not attend college but did complete high school, his scores were compared to students in grade 12. MM's scores were compared with those in his grade (grade $10)$.

The Thematic Apperception Test (TAT; Murray, 1938, 1943) was used as a test of the ability to recognise social situations and to formulate reasonable narrative scenarios. Subjects were shown six of the cards (cards 1, 2, 6BM, $8 \mathrm{BM}, 12 \mathrm{M}$, and $13 \mathrm{MF}$ ) and were requested to generate a story with a beginning, middle, and end, and an explanation of what the characters are thinking, feeling, and doing. TAT responses were not evaluated in the normal clinical manner for psychopathology, but were rated with regard to level of logic, complexity of social understanding, and insight regarding the content commonly mentioned for each card.

The Rorschach Inkblot Test (Rorschach, 1942) was administered as another probe of conventional visual recognition and interpretation of ambiguous information. The results were scored according to Exner's Comprehensive System (1990). Subscores were computed on the Rorschach Interpretative Assessment Procedure (RIAP) computer programme. All analyses presented here were based on the structural summary scores and age-appropriate normative data provided by Exner (1990) and interpretation of structural summary scores was based on Exner (1991). The Exner system imposes an objective set of rules on the scoring and interpretation of Rorschach responses. In order to examine our hypotheses about impaired social understanding and emotional processing, the scores on the capacity for control/coping, affect, and cognitive clusters were analysed. We also report any significant scores on the clinical indices, although it is uncertain whether the Exner labels for these indices are at all appropriate in the case of ACC.

The Child Behavior Checklist (CBCL; Auchenbach, 1991) contains a list of behavioural problems and competencies which are rated separately by a parent and by the child. The CBCL provides scales of Activity, Social, and School competencies, as well as subscales for Behavioral Problems (Withdrawal, Somatic Complaints, Anxiety/Depression, Social Problems, Thought Problems, Attention Problems, Delinquent Behavior, and Aggression). Age appropriate norms were used to derive $T$-scores for each scale. 


\section{Procedures}

The tests were administered during three 3 to 4 hour sessions. A variety of tests were administered during each session and breaks were allowed as necessary to avoid interference or fatigue effects. The WISC-R and the Child Behavior Checklists (parent report and youth report form) were completed for both individuals approximately 18 months prior to the remainder of the testing reported herein. Neither subject appeared to have changed in any noteworthy way in intelligence or behaviour during this period.

The Rorschach and TAT were administered and scored by an experienced test administrator who was a stranger to the two patients. The Rorschach was then scored by another experienced clinician who was blind to the diagnosis of the subject. For scoring and normative comparison on the TAT, the stories produced by the two subjects were mixed in with eight other TAT response protocols of adolescent males in similar age range (14-22 years). The 10 protocols were independently ranked from best to worst on the aforementioned categories (logic, complexity, and insight) by two individuals experienced with the TAT and blind as to the identity of the 10 protocols. A cumulative rank was determined for each subject by summing their rankings across the three categories. Individual performance was evaluated based on the probability of being at or below the ordinal position of the cumulative rank of one rater (e.g., 10th position, $p=.10$ ) multiplied by the probability of attaining the given rank of the other rater. Thus, the probability of a subject getting the lowest cumulative rank from both raters by chance would be $p=.01$ (i.e., $.1 \times .1$ ).

The intention of this research was to describe clinically significant deficits in these two normally intelligent ACCs with respect to normal peers within the context of a clinical neuropsychological assessment. Thus, the results of tests of ACC patients in this study were compared to published norms where relevant norms were available. Although the issue of appropriate control subjects for studies of ACC has generated some controversy (e.g., Cook, 1990; Lassonde \& Bryden, 1990; Lassonde, Bryden, \& Demers, 1990), this issue is not as acute when one is studying ACC patients with normal intelligence.

For Letter and Number Series normative data are not available. Therefore, results of JD and MM were compared to the mean and standard deviation of a control group of 17 normal young adults (age range $=18-36$ years, mean $=$ 22.47, $\mathrm{SD}=4.53$ ) with IQs that span the range of the two ACC individuals (FSIQ: range $=84-107$; mean $=94.24, \mathrm{SD}=6.49$ ).

\section{Statistics}

Results for all tests on which norms are available are reported as z-scores, allowing for cross-test comparisons. In order to enhance the qualitative descriptions of the neuropsychological and psychosocial performance of these 
two ACC patients, joint probabilities of test outcomes were calculated as an index of common departures from normative samples. Z-scores were used to determine the probability of each particular score for each subject (i.e., the probability that an observation would be this deviant from the normative mean, presuming random sampling). In summarising results for the two cases, if $z$ scores were either both above .50 or both below - .50 , then the significance of a finding was accepted as the joint probability of the $z$-scores of the two acallosals. This statistical procedure was appropriate for data summaries given the case study nature of this research.

\section{RESULTS}

\section{Cognitive performance}

Psychometric Intelligence. Both subjects had FSIQs within normal limits and PIQ over 1.5 SD higher than VIQ (MM: FSIQ = 108, VIQ=97, PIQ=114; JD: FSIQ=87, VIQ=80, PIQ=96). On verbal subtests, MM's scores ranged from 6 to 11 and JD's ranged from 6 to 9. On performance subtests, MM's scores ranged from 9 to 15 and JD's scores ranged from 8 to 13 (see Figure 1).

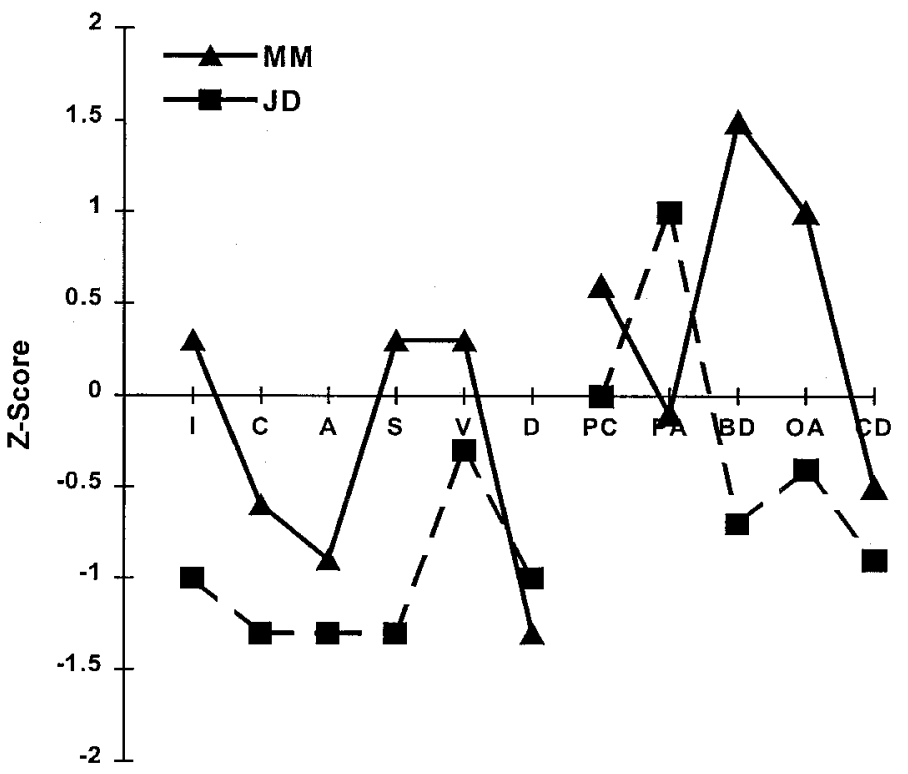

VIQ

PIQ

Figure 1. WISC-R profiles for MM at age 14 (solid line) and JD at age 16 (dashed line). Subscale abbreviations are: Information (I), Comprehension (C), Arithmetic (A), Similarities (S), Vocabulary (V), Digit Span (D), Picture Completion (PC), Picture Arrangement (PA), Block Design (BD), Object Assembly (OA), and Coding (CD). 
Overall, their subtest profiles did not show a consistent pattern, with the exception of both having scores over 1 SD below the mean on Arithmetic and Digit Span, and both having their lowest performance score in Coding (Digit Symbol) which was nevertheless within the normal range.

Wide Range Achievement Test. Percentile scores on the WRAT-3 were converted to $z$-scores for comparison (Table 1). Although MM was clearly deficient in spelling, there was no evidence of consistent problem in either reading or spelling. However, both individuals scored at the 5th percentile in math.

Raven's Colour Progressive Matrices. On the RCPM, MM performed in the low end of the average range and JD's performance was significantly impaired, resulting in a significant joint probability of deficit on this problemsolving measure (Table 1).

Tactual Performance Test. On the 10-block TPT, both MM and JD performed poorly with the dominant hand, resulting in a significant joint probability (see Table 1). However, they performed markedly worse on the subsequent attempt with the nondominant hand $(z=-8.08 ; z=-9.75)$, indicating an additional deficit in interhemispheric (interhand) sharing of the spatial information regarding the formboard. On the less complex 6-block version, MM and JD scored in the normal range (with respect to norms for 13-

TABLE 1

Results of tests of cognitive ability: Z-scores

\begin{tabular}{llll}
\hline Test & $M M$ & $J D$ & Joint probability \\
\hline WISC IQ & FSIQ $Z=.53$ & FSIQ $Z=-.86$ & n.s. \\
& VIQ $Z=-.20$ & VIQ $Z=-1.3$ & n.s. \\
& PIQ $Z=.93$ & PIQ $Z=-.27$ & n.s. \\
WRAT-3 & Reading $Z=-.74$ & Reading $Z=.67$ & n.s. \\
& Spelling $Z=-2.30$ & Spelling $Z=-.37$ & n.s. \\
& Math $Z=-1.66$ & Math $Z=-1.66$ & $p<.01$ \\
Raven's CPM & $Z=-.72$ & $Z=-2.32$ & $p<.01$ \\
TPT: 6-block & DH: $Z=0.80$ & DH: $Z=0.40$ & n.s. \\
(13 yr norms) & NDH: $Z=-0.29$ & NDH: $Z=0.57$ & n.s. \\
TPT: $10-$ block & DH: $Z=-5.35$ & DH: $Z=-2.00$ & $p<.001$ \\
& NDH: $Z=-8.08$ & NDH: $Z=-9.75$ & $p<.0001$ \\
Category Test & $Z=-1.2$ & $Z=-2.2$ & $p<.001$ \\
Letter Series* & $Z=-1.83$ & $Z=-2.69$ & $p<.001$ \\
Number Series* & $Z=-1.16$ & $Z=-2.01$ & $p<.01$ \\
Proverbs Test & $Z=-1.0$ & $Z=-0.7$ & $p<.05$ \\
\hline
\end{tabular}


year-olds), with much less relative difference between dominant and nondominant hand performance (Table 1).

Category Test. Category test performance was low average in MM and markedly impaired in JD, resulting in significant joint probability of deficit (see Table 1). For both individuals, a large portion of their errors occurred on the third subtest, with JD also making a large number of errors on subtests four and five. This overall performance suggests difficulty in recognition of abstract categories of stimuli presented.

Letter and Number Series. On the number series test, MM's performance was low average and JD's performance was significantly impaired $(z=-.1 .6$; $z=-2.01$; see Table 1). On the letter series, they both exhibited greater impairment $(z=-1.83 ; z=-2.69)$. Thus, they exhibited a significant joint probability of deficit on both tests.

Proverbs Test. Neither MM nor JD were able to generate any appropriate answers to the free answer portion of the proverbs test, but both attained a low average abstraction score on the best-answer form (still resulting in a significant joint probability). Thus, although they could both correctly identify the meaning of some proverbs (albeit still significantly below expectation), they could not independently generate an appropriate abstract verbal meaning.

\section{Psychosocial performance}

MMPI-2. On the MMPI-2 both subjects exhibited L-scale elevations $(\mathrm{T}=83 ; \mathrm{T}=66$; joint probability: $.021[\mathrm{MM}] \times .110[\mathrm{JD}]=p<.001)$ and low scores on most other scales (see Figure 2). An elevated L-scale with low values on other scales indicates significant psychological naiveté, unsophisticated defenses, and poor self-understanding. JD had no other elevations, but MM had a notable elevation on scale 9 (mania, $\mathrm{T}=69$ ), which would indicate that MM has higher levels of energy than JD (a result consistent with our behavioural observations).

TAT. JD placed second to last and MM placed last in the rankings of TAT stories. Both gave inadequate logic for their stories, lacked complexity of social understanding, and missed the content commonly elicited by the cards (joint probability: $.01[\mathrm{MM}] \times .02[\mathrm{JD}]=p<.01)$. This finding is consistent with the naivety evident on the MMPI-2, and suggests that these acallosals lack insight into the complexities of social behaviour as indicated in the pictures.

Rorschach. On the Rorschach, MM and JD had several consistent findings (joint probabilities, $p<.001$, see Table 2), including deficits in interest in, and organisation of, emotional stimulation (Affective Ratio, Modulated Emotion), perceptual distortions (Distorted Form), lack of conventional form perception (Popular Responses, Conventional Form), and lowered sense of social/circumstantial efficacy (Inanimate Movement). The scores indicate that both subjects: (1) have some deficits in psychosocial coping; (2) 


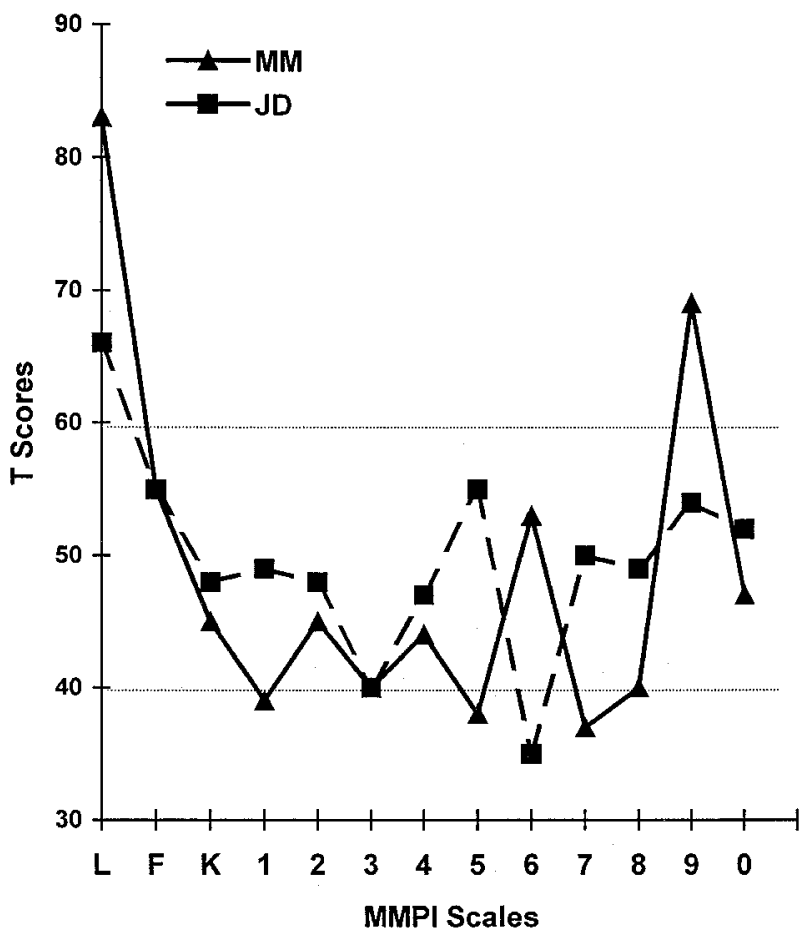

Figure 2. MMPI-2 scores of JD (dashed line), and MM (solid line). The scale descriptors are: L, Lie; F, Infrequency; K, Correction, 1, Hypocondriasis; 2, Depression; 3, Conversion Hysteria; 4, Psychopathic Deviate; 5, Masculinity/Feminity; 6, Paranoeia; 7, Psychasthenia; 8, Schizophrenia; 9, Hypomania; 10, Social Introversion.

tend to misinterpret or avoid emotional information; and (3) have impairments in the cognitive processes of interpreting and assigning meaning to ambiguous stimuli. The subjects varied in the cluster indexes for which they qualified, indicating that other aspects of their individual response patterns were somewhat unique.

Child Behavior Checklist. Absence of obvious psychopathology or unusual behaviour was supported by the Youth Self Report and the Parent Report on the CBCL. Results of the CBCL do not indicate marked behavioural problems in either of these adolescents with ACC (see Figure 3). Both young men experienced, and their parents recognised, some problems in school performance $(\mathrm{MMT}=33$; JDT $=25)$. MM and, most particularly, JD reported an unusually high number of somatic complaints, which would be consistent with an alexithymic profile.

Behavior Observation. Behaviour of both subjects in the laboratory was cooperative and friendly, but in both cases appeared immature for their 
Exner Scoring of the Rorschach: T-scores

\begin{tabular}{lcc}
\hline & \multicolumn{2}{c}{ Subjects } \\
\cline { 2 - 3 } Scores & $M M$ & $J D$ \\
\hline Psychosocial coping & & \\
Experience Actual (EA) & 61.45 & $14.08^{*}$ \\
Experience Base(es) & 46.59 & 49.33 \\
Stress Tolerance (D) & 39.57 & $31.11^{+}$ \\
Optimal Stress Tolerance (Adjusted D) & $28.97^{+}$ & 36.21 \\
Human Movement Active (Ma) & $66.80^{+}$ & $30.88^{+}$ \\
Human Movement Passive (Mp) & 65.27 & 36.06 \\
Animal Movement (FM) & 39.48 & 60.92 \\
Inanimate Movement (m) & $73.46^{+}$ & $72.12^{+}$ \\
Emotional processing & & \\
Affective Ratio (Afr)** & 35.00 & $22.50^{+}$ \\
Modulated Emotion (FC)** & $31.33^{+}$ & $28.24^{+}$ \\
Unmodulated Emotion (CF) & 44.44 & 39.29 \\
Cognitive processing & & \\
Popular Responses (P)** & $31.06^{+}$ & $7.63^{*}$ \\
Pairs (2) & 64.50 & $14.28^{*}$ \\
Conventional Form (X+\%)** & $17.14^{*}$ & $17.50^{*}$ \\
Conventional Pure Form (F+\%) & 48.89 & 33.53 \\
Distorted Form (X - \%)** & $100.00^{*}$ & $102.00^{*}$ \\
Unusual Form (Xu \%) & 50.00 & 48.57 \\
Special Scores (W Sum 6) & 56.88 & 62.87 \\
\hline
\end{tabular}

${ }^{+}$Difference from $T=50, p<.05$. * Difference from $T=50, p<.001 . * *$ Joint probability, $p<.001$.

chronological age. MM frequently made jokes that were concrete and circumstantial and he tended to perseverate on them. Both subjects worked diligently and rarely took breaks between tests.

\section{DISCUSSION}

We have studied the cognitive and psychosocial function of two older adolescent males with agenesis of the corpus callosum and normal range IQ in order to test a particular hypothesis regarding the relationship between callosal absence, cognitive deficits, and psychosocial disabilities. The primary assumption of this hypothesis is that callosal absence results in a reduction of connectivity between cortical processing systems available for higher cognitive information processing. We hypothesised that the consequences of this reduction in interconnectivity would not be apparent in performance on more overlearned cognitive tasks (i.e., WAIS IQ may well be normal). However, 


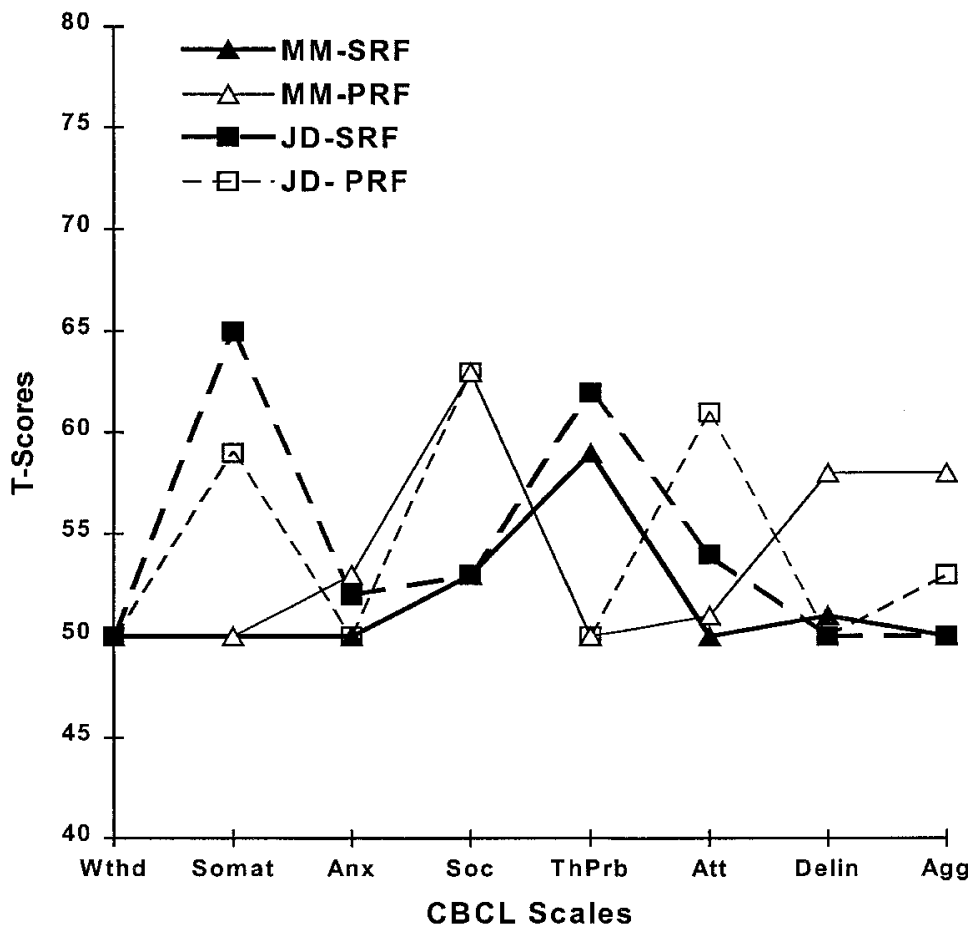

Figure 3. CBCL Behavior Problem Scales of MM (solid lines) and JD (dashed lines), both Self Report Form (SRF; thick lines) and Parent Report Form (PRF; thin lines). Scales correspond with the following factors: withdrawn (Wthd); somatic complaints (Somat); anxious/depressed (Anx); social problems (Soc); thought problems (ThtPr); attention problems (Att); delinquent behaviour (Delin); aggressive behaviour (Agg).

more novel and complex tasks that put a high demand on reasoning, concept formation, and problem-solving ability will result in deficits which are more severe than would be predicted based on IQ scores. This distinction between realms of cognitive functions is consistent with the differences between crystallised and fluid intelligence (Cattell, 1963; Horn, 1968), or between convergent and divergent thinking (Guilford, 1967). Finally, we hypothesised that understanding social situations and their psychological and interpersonal significance would be particularly difficult for ACC individuals, as these situations are often complex and relatively novel. Thus, although routine social behaviour may well be normal, the subtleties of situations involving more complex social relationships will be missed. According to this theory, we would not necessarily expect more serious forms of psychopathology or behavioural disorder. 


\section{Cognitive abilities}

Both of our subjects have full-scale IQ scores within the normal range $(\mathrm{MM}=108, \mathrm{JD}=87)$. This indicates that these individuals with ACC have normal "crystallised intelligence" (i.e., they are capable of normal levels of performance on more routinised, overlearned cognitive processing tasks). Although there are no subscales that were markedly abnormal for both, there is a tendency for low scores on Arithmetic, Digit Span, and Coding, which are subtests where some amount of fluid intelligence is required. Despite the fact that ACC is relatively rare, such individuals with IQs within the normal range have been reported numerous times in the literature. Chiarello (1980) summarised 19 cases of complete ACC with IQs above 70 which had appeared in the literature at that time, 14 of these with IQs above 80, and 7 with scores above 90. Sauerwein et al. (1994) reported another 17 cases that had appeared after 1980, of which 13 had IQs above 80, and eight above 90. Although both of the individuals reported herein have higher Performance than Verbal IQ, Sauerwein et al. make it clear that there is no trend for individuals with ACC to have large Verbal-Performance differences, or to have one domain consistently better than the other. Regarding specific WISC/WAIS subscales, Sauerwein et al. report lowest performance on Digit Symbol (Coding) and Arithmetic, a pattern of subscale performance seen to some degree in our two acallosals.

The pattern of performance on the WRAT-3 subtests of academic achievement again suggest greatest problems in more fluid aspects of intelligence. Neither individual was outside the normal range in reading, and only MM had difficulty in spelling. Both ACCs had difficulty with math to a degree not predictable from their FSIQ. Math tests require problem solving.

Performance on the Raven's Coloured Progressive Matrices does not appear consistent between the two individuals. However, the difference in $z$-scores between Raven's and FSIQ differences $(\mathrm{MM}=1.25$; JD=1.46) does suggest a consistent deficit on the Raven's. The fact that the Raven's test is considered a more "culture fair' ' test of general intelligence also indicates that it is a test not heavily influenced by prior learning and experience.

The TPT provides data with reference to both spatial concept formation (i.e., original performance with the dominant hand) and capacity for transfer of tactile-spatial learning for both simple (6-block) and more complex (10-block) versions of this task. Both MM and JD were normal in dominant hand performance on the 6-block version, but relatively poor in 10-block dominant hand TPT performance. This suggests deficiencies in concept formation that are only apparent with more complex tasks. Interhand transfer of the spatial learning (nondominant hand performance) was normal for the 6-block version. This result is consistent with the report of Sauerwein et al. (1981). However, we found clear deficits in interhand transfer in both subjects using the 10-block version of the TPT. For the 10-block version, time to solve the puzzle increased 
rather than decreased for the nondominant hand. Previous research has also described both moderate deficits in dominant hand TPT performance, and relatively greater deficits in the subsequent attempt with the nondominant hand (Ferriss \& Dorsen, 1975; Fischer et al., 1992; Gott \& Saul, 1978; Jeeves, 1979; Russell \& Reitan, 1955; Sauerwein et al., 1994; Solursh et al., 1965).

Results of the Categories Test also support the hypothesis that concept formation and problem solving are worse than would be predicted by their intelligence levels (i.e., MM and JD had scores at the 12th and 1st percentiles, respectively). Qualitative analysis of Categories performance indicates that they had the greatest difficulty in distinguishing and responding to the location of the "different" geometric form or colour (Subtest III), particularly after successfully solving problems based on counting or numbers (Subtest I and II). Response latencies were longer for incorrect than correct-response trials for both subjects, suggesting that they were not responding incorrectly because they were impulsive.

Like the Raven's and the Categories Test, the letter and number series tasks require reasoning, concept formation, and a form of problem solving, and both ACCs showed evidence of deficit. These tasks require the individual to recognise and abstract a pattern in the previous examples and apply the pattern to complete the last letter or number of the series.

The hypothesis of diminished reasoning and concept formation (i.e., deficits in fluid intelligence and divergent thinking) is also supported by results of the Proverbs Test. Here, difference in level of demand between the two versions graphically illustrates the nature of deficits in ACC. MM and JD had significant difficulty independently producing metaphoric interpretations from the proverbs, but could recognise appropriately such abstract meanings when given multiple choices. Two interpretations might be offered for this pattern of results. First, it may be that a rich and continuous interhemispheric interaction between linguistic analysis and visual imagery is necessary in understanding a proverb in the absence of specific choices. Alternatively, if the disability lies in an inability to verbalise their understanding, the problem may be a more specific deficit in transfer from the right hemisphere, which may hold the semantic networks needed to understand proverbs, to the left hemisphere which is responsible for speech. In both cases, the difficulty would be due to diminished interaction between the processing systems or modules that are necessary for self-generation of proverb meanings. Correct recognition of proverb meaning might be possible on the basis of left hemisphere processing (i.e., without efficient interhemispheric exchange).

\section{Psychosocial abilities}

The hypothesised impact of these cognitive processing deficits on social understanding is indicated by deficits in self-perception indicated on the MMPI-2. Both MM and JD had significantly elevated L (lie) scale scores, but 
nearly all the other scales fell below the normal range. Although this may indicate an attempt to "fake good", it is more likely an indication of honest responses lacking in self-awareness. This is consistent with the strong subjective impression one has of both individuals as somewhat unsophisticated in their self-awareness and naively incapable of guile and pretence. Thus, the deficits of ACC individuals in concept formation would appear to impact the level of sophistication of their self-perception.

Difficulty in recognising and processing complex socioemotional material was evident on the TAT. Both MM and JD were unable to generate a reasonable story from the TAT pictures. They were both rated at the bottom of a peer group with respect to the appreciation of common content, as well as the logic and complexity of their stories. The fact that MM and JD both tended to overlook the obvious social implications of the pictures that are commonly identified by most subjects may indicate that ACC patients have a disability in comprehension of complex social dynamics and in appreciation of the subtle nuances of social situations. MM and JD were particularly unable to describe the emotional implications of the TAT pictures, responding (as would an alexithymic) as if they were completely unaware of the emotional content in the cards. However, most alexithymic individuals would nevertheless be able to generate coherent stories, which these two ACC subjects could not.

As with the Proverbs Test and the TAT, the most consistent deficits in responses to the Rorschach Inkblot cards were found in the nature and sophistication of the responses generated. Both ACC subjects exhibited deficits in perceptual/interpretive accuracy, lack of conventionality in responses, lack of verbalisation of emotional content, and a tendency toward faulty judgement. These abnormalities resulted in profiles suggestive of individuals who tend to be unusual in how they perceive novel or ambiguous stimuli, and consequently tend to reach unconventional conclusions. It is possible that their odd interpretations of the ink blots are related to an inability to articulate their imaginative and emotional responses, which leaves them with fewer interpretive options than the normal population.

Deficits in interhemispheric transfer suggestive of callosal dysfunction have been found in individuals with various "behavioural" difficulties, such as schizophrenia and schizoaffective disorders (David, 1989; Gulmann, Wildschiodtz, \& Orbaek, 1982; Hatta, Yamamoto, \& Kawabata, 1984; Njiokiktjien, 1991), attention deficit hyperactivity disorder (Giedd et al., 1994; Hynd et al., 1991), and autism (Belmonte et al., 1995). For example, schizophrenic patients showed clear deficits as compared to normals and schizoaffective patients on tests of callosal transfer (Craft, Willerman, \& Bigler, 1987; Raine et al., 1990). Although interhemispheric transfer deficits have been found in the aforementioned disorders, dysfunction in interhemispheric communication has not, to this point, been deemed either a critical diagnostic criterion or an important aetiological factor in any of these forms of psychopathology. 
The Rorschach results in these ACC patients offer support to the hypotheses that ACC patients exhibit abnormalities in cognitive processing, as indexed by markedly abnormal scores indicating unusual thoughts and unconventional perceptions (Distorted Forms, Conventional Forms, and Popular Responses, see Table 2). There is also some indication of alexithymia-like symptoms in their ability to verbally organise emotions (Affective Ratio, Modulated Emotions). MM actually met the Exner criteria for the schizophrenia, depression, and hypervigilance indices, and JD's tendency toward distorted interpretation and faulty judgement was consistent with schizophrenia-like symptomatology within the Exner system. However, behavioural observations of both MM and JD were not at all consistent with these diagnoses. Neither subject gave particularly bizarre responses and neither have ever reported hallucinations or delusions. Thus, the Rorschach results would not be related to schizophrenia, but rather indicative of an unconventional thought process which leads to schizophrenic-like responses within the context of responding to Rorschach stimuli. Thus, the diagnostic label "schizophrenia" would be inappropriate when the aetiology of the unusual Rorschach responses is known to be ACC. JD exhibited significant deficits in available resources (Experience Actual) and self-awareness (Pairs). These scores indicate deficits in psychosocial coping and personal awareness.

Problems in self-awareness and social understanding were evident in the results of the MMPI-2 and TAT, as well as the somewhat unusual responses indicated on the Rorschach. However, the Child Behavior Checklist results for both of our ACC subjects did not suggest psychopathology or grossly deviant overt social behaviour. Nevertheless, on the CBCL both subjects rated themselves high on Thought Problems (admitting to certain unusual thoughts), but low on Social Problems. In contrast, both subjects' mothers rated the young men high on Social Problems and low on Thought Problems. One source of this difference in ratings between subjects and the mothers may be the naivety and lack of self-perception of the acallosals suggested in the MMPI-2, leaving them unaware of social problems recognised by parents. However, unusual thoughts might not be recognised by parents, particularly if the acallosals have difficulty giving spontaneous verbal expression to inner thoughts and experiences. Lack of emotional communication was found to be characteristic of both complete and partial callosal agenesis in O'Brien's (1994) survey of parents of acallosal children.

Although parts of the syndrome of deficits we have described are reflected in various places in the existing literature, there is at least one other complete description of an ACC patient that includes sufficient cognitive and psychosocial evaluation for comparison. Rourke (1989) describes the cognitive and psychosocial consequences of ACC in an early adolescent female called "Mary'. Mary was tested at 9 and 11.5 years of age, achieving FSIQ values of 94 and 78, respectively. Among the most marked neurocognitive deficiencies 
was TPT performance, with $T$-scores below 10 for both dominant and nondominant hands. She also showed clear deficits on the Categories test $(T<30)$. Thus, Mary had significant deficiencies in concept formation and problem solving in novel and complex situations. Mary was also deficient in social relationships. On the Personality Inventory for Children (an inventory filled out by a parent), Mary deviated significantly from normal on indices of social skills, psychosis, somatic concerns, and overall adjustment. On the Vineland Adaptive Battery, she manifested clear problems in communication, daily living, and socialisation. Rourke presents Mary as an illustration of a nonverbal learning disability. However, all ACC individuals do not fit Rourke's definition of a nonverbal learning disability. Nevertheless, the case of Mary supports our theory of a relationship between callosal agenesis, deficits in complex reasoning and problem solving, and psychosocial functioning.

Case studies such as these do not allow final conclusions regarding the relationships we have hypothesised. However, both reports in the literature, and the profiles of JD and MM provide evidence in favour of a syndrome of deficits in ACC - reduced ability for reasoning, concept formation, and problem solving, and deficits in complex psychosocial perception, understanding, and ageappropriate social behaviour. We believe that diminished interaction between bihemispheric processing systems is at the root of this syndrome.

Manuscript received 10 November 1998 Revised manuscript received 15 November 1999

\section{REFERENCES}

American Psychological Association. (1992). Ethical principles of psychologists and code of conduct. American Psychologist, 47, 1597-1611.

Auchenbach, T.M. (1991). The child behavior checklist. Burlington, VT: University Associates in Psychiatry.

Belmonte, M., Egaas, B., Townsend, J., \& Courchesne, E. (1995). NMR intensity of corpus callosum differs with age but not with diagnosis of autism. Neuroreport, 6, 1253-1256.

Bogen, J.E., \& Bogen, G.M. (1969). The other side of the brain. III: The corpus callosum and creativity. Bulletin of the Los Angeles Neurological Society, 34, 191-202.

Brown, W.S., Jeeves, M.A., Dietrich, R., \& Burnison, D.S. (1999). Bilateral field advantage and evoked potential interhemispheric transmission in commissurotomy and callosal agenesis. Neuropsychologia, 37, 1154-1180.

Buchanan, D.C., Waterhouse, G.J., \& West, S.C. (1980). A proposed neurophysiological basis of alexithymia. Psychotherapy and Psychosomatics, 34, 248-255.

Cattell, R.B. (1963) Theory of fluid and crystallized intelligence: A critical experiment. Journal of Educational Psychology, 54, 1-22.

Chiarello, C. (1980). A house divided? Cognitive functioning with callosal agenesis. Brain and Language, 11, 128-158.

Cioni, G., Bartalena, L., Biagioni, E., \& Boldrini, A. (1994). The normal, absent and abnormal corpus callosum: Postnatal sonographic findings. In M. Lassonde \& M.A. Jeeves (Eds.), Callosal agenesis: A natural split brain? (pp.69-76). New York: Plenum. 
Cook, N.D., Brugger, P., Regard, M., \& Landis, T. (1990). On the role of the corpus callosum in cerebral laterality: A comment on Lassonde, Bryden, and Demers. Brain and Language, 39, 471474.

Craft, S., Willerman, L., \& Bigler, E.D. (1987). Callosal dysfunction in schizophrenia and schizoaffective disorder. Journal of Abnormal Psychology, 96, 205-213.

David, A. (1989). Reading about the split-brain syndrome. British Journal of Psychiatry, 154, 422425.

Dennis, M. (1981). Language in a congenitally acallosal brain. Brain and Language, 12, 33-53.

Exner, J.E., Jr. (1990). A Rorschach workbook for the comprehen sive system (3rd ed.). Asheville, NC: Rorschach Workshops.

Exner, J.E., Jr. (1991). The Rorschach: A comprehensive system (2nd ed.), Vol. 2). New York: Wiley-Interscience.

Ferriss, G.S., \& Dorsen, M.M. (1975). Agenesis of the corpus callosum: 1. Neuropsychological studies. Cortex, 11, 95-122.

Fischer, M., Ryan, S.B., \& Dobyns, W.B. (1992). Mechanisms of interhemispheric transfer and patterns of cognitive function in acallosal patients of normal intelligence. Archives of Neurology, 49, 271-277.

Giedd, J.N., Castellanos, F.X., Casey, B.J., Kozuch, P., King, A.C., Hamburger, S.D., \& Rapoport, J.L. (1994). Quantitative morphology of the corpus callosum in attention deficit hyperactivity disorder. American Journal of Psychiatry, 151, 665-669.

Gorham, D.R. (1956). A proverbs test for clinical and experimental use. Psychological Reports, 2, 112.

Gott, P.S., \& Saul, R.E. (1978). Agenesis of the corpus callosum: Limitations of functional compensation. Neurology, 28, 1272-1279.

Guilford, J.P. (1967). The nature of human intelligence. New York: McGraw-Hill.

Gulmann, N.C., Wildschiodtz, G., \& Orbaek, K. (1982). Alteration of hemispheric conduction through corpus callosum in chronic schizophrenia. Biological Psychiatry, 17, 585-594.

Halstead, W.C. (1947). Brain and intelligence. Chicago, IL: University of Chicago Press.

Hathaway, S.R., \& McKinley, J.C. (1989). MMPI-2: Minnesota multiphasic personality inventory- 2. Minneapolis, MN: University of Minnesota Press.

Hatta, T., Yamamoto, M., \& Kawabata, Y. (1984). Functional hemispheric differences in schizophrenia: Interhemispheric transfer deficit or selective hemisphere dysfunction? Biological Psychiatry, 19, 1027-1036.

Heaton, R.K., Grant, I., \& Matthews, C.G. (1991). Comprehen sive norms for an expanded HalsteadReitan Battery: Demographic corrections, research findings, and clinical applications. Odessa, FL: Psychological Assessment Resources.

Hoppe, K.B., \& Bogen, J.E. (1977). Alexithymia in 12 commissurotomized patients. Psychotherapy and Psychosomatics, 28, 148-155.

Horn, J.L. 91968). Organization of abilities and the development of intelligence. Psychological Review, 75, 242-259.

Hynd, G., Semrud-Clikeman, M., Lorys, A., Novey, E., Eliopulos, D., \& Lyytinen, H. (1991). Corpus callosum morphology in attention deficit hyperactivity disorder: Morphometric analysis of MRI. Journal of Learning Disabilities, 24, 141-146.

Jastak, S., \& Wilkinson, G. (1984). The wide range achievement test: Administration manual. Wilmington, DE: Jastak Association.

Jeeves, M.A. (1979). Some limits to interhemispheric integration in cases of callosal agenesis and partial commissurotomy. In I. Russell, M. van Hof, \& jG. Berluchhi (Eds.), Structure and function of the cerebral commissures (pp.449-474). New York: Macmillan.

Jeeves, M.A., \& Milner, A.D. (1987). Specificity and plasticity in interhemispheric integration: Evidence from callosal agenesis. In D. Ottoson (Ed.), Wenner-Gren International Symposium Series: Vol 47. The duality and unity of the brain: Unified functioning and specialization of the hemispheres. Basingstoke, UK: Macmillan. 
Jeeves, M.A., \& Temple, C.M. (1987). A further study of language function in callosal agenesis. Brain and Language, 32, 325-335.

Khanna, S., Chugani, H.T., Messa, C., \& Curran, J.G. (1994). Corpus callosum agenesis and epilepsy: PET findings. Pediatric Neurology, 10, 221-227.

Lassonde, M., \& Bryden, M.D. (1990). Dichotic listening, callosal agenesis and cerebral laterality. Brain and Language, 39, 475-481.

Lassonde, M., Bryden, M.D., \& Demers, P. (1990). The corpus callosum and cerebral speech lateralization. Brain and Language, 38, 195-206.

Lesser, I.M. (1981). A review of the alexithymia concept. Psychosomatic Medicine, 43, 531-543.

Murray, H.A. (1938). Explorations in personality. New York: Oxford University Press.

Murray, H.A. (1943). Thematic Apperception Test Manual. Cambridge, MA: Harvard University Press.

Nemia, J.C., \& Sifneos, P.E. (1970). Affect and fantasy in patients with psychosomatic disorder. In O.W. Hill (Ed.), Modern trends in psychosomatic medicine: Vol. II. New York: Appleton, Century, Crofts.

Njiokiktjien, C. (1991). A historical perspective. In G. Ramaekers \& C. Njiokiktjien (Eds.), The child's corpus callosum. Amsterdam: Suyi.

Njiokiktjien, C., \& Ramaekers, G. (1991). Developmental interhemispheric disconnection and learning disabilities. In G. Ramaekers \& C. Njiokiktjien (Eds.), The child's corpus callosum. Amsterdam: Suyi.

O'Brien, G. (1994). The behavioral and developmental consequences of corpus callosal agenesis and Aicardi Syndrome. In M. Lassonde \& M. Jeeves (Eds.), Callosal agenesis: A natural split brain? (pp.235-246). New York: Plenum.

Paul, A.L., \& Brown, W.S. (1996). Psychosocial deficits in agenesis of the corpus callosum [abstract]. Journal of the International Neuropsychological Society, 2, 41.

Raine, A., Harrison, G., Reynolds, F., Shear, C., Cooper, J., \& Medley, I. (1990). Structural and functional characteristics of the corpus callosum in schizophrenics, psychiatric controls, and normal controls. Archives of General Psychiatry, 47, 1060-1064.

Raven, J.C. (1965). Guide to using the colored progressive matrices. London: H.K. Lewis.

Raven, J.C., Court, J., \& Raven. (1976). Manual for Raven's progressive matrices. London: H.K. Lewis.

Reitan, R.M., \& Davison, L.A. (Eds.) (1974). Clinical neuropsychology: Current status and applications. Washington, DC: Winston.

Rorschach, H. (1942). Psychodiagnostics: A diagnostic test based on perception (P. Lemkau \& B. Kronenber, Trans.). Bern: Huber. (Original work published 1921)

Rourke, B.P. (1989). The nonverbal learning disabilities: The syndrome and the model. New York: Guilford Press.

Russell, J.R., \& Reitan, R.M. (1955). Psychological abnormalities in agenesis of the corpus callosum. Journal of Nervous and Mental Disease, 121, 205-214.

Sanders, R.J. (1989). Sentence comprehen sion following agenesis of the corpus callosum. Brain and Language, 37, 59-72.

Sauerwein, H.C., Lassonde, M., Cardu, B., \& Geoffroy, G. (1981). Interhemispheric integration of sensory and motor functions in agenesis of the corpus callosum. Neuropsychologia, 19, 445454.

Sauerwein, H.C., Nolin, P., \& Lassonde, M. (1994). Cognitive functioning in callosal agenesis. In M. Lassonde \& M.A. Jeeves (Eds.), Callosal agenesis: A natural split brain? (pp.221-233). New York: Plenum.

Schaie, K.W. (1983). The Seattle Longitudinal Study: A twenty-one year exploration of psychometric intelligence in adulthood. In K.W. Schaie (Ed.), Longitudinal studies of adult cognitive development (pp.64-135). New York: Guilford Press.

Schaie, K.W., Willis, S., \& O'Hanlon, A. (1994). Perceived intellectual performance change over seven years. Journal of Gerontology: Psychological Sciences, 49, 108-118. 
Sifneos, P.E. (1972). Short-term psychothe rapy and emotional crisis. Cambridge, MA: Harvard University Press.

Sifneos, P.E. (1973). The prevalence of "alexithymic" characteristics in psychosomatic patients. Psychotherapy and Psychosomatics, 22, 255-262.

Solursh, L.P., Margulies, A.I., Ashem, B., \& Stasiak, E.A. (1965). The relationship of agenesis of the corpus callosum to perception and learning. Journal of Nervous and Mental Disease, 141, 180189.

Temple, C.M., \& Ilsley, J. (1994). Sounds and shapes: Language and spatial cognition in callosal agenesis. In M. Lassonde \& M.A. Jeeves (Eds.), Callosal agenesis: A natural split brain? New York: Plenum.

Temple, C.M., Jeeves, M.A., \& Vilarroya, O. (1989). Ten pen men: Rhyming skills in two children with callosal agenesis. Brain and Language, 37, 548-564.

Temple, C.M., Jeeves, M.A., \& Vilarroya, O. (1990). Reading in callosal agenesis. Brain and Language, 39, 235-253.

TenHouten, W.D., Hoppe, K.B., Bogen, J.E., \& Walter, D.O. (1985). Alexithymia and the split-brain: IV. Gotteschalk-Gleser content analysis, an overview. Psychotherapy and Psychosomatics, 44, 113-121.

TenHouten, W.D., Walter, D.O., Hoppe, K.B., \& Bogen, J.E. (1987). Alexithymia and the split brain: V. EEG alpha band interhemispheric coherence analysis. Psychotherapy and Psychosomatics, 47, $1-10$.

TenHouten, W.D., Walter, D.O., Hoppe, K.B., \& Bogen, J.E. (1988). Alexithymia and the split brain. VI. EEG correlates of alexithymia. Clinical Psychiatry, 11, 317-329.

Thurstone, L.L., \& Thurstone, T.B. (1949). Examiner manual for the SRA primary mental abilities test. Chicago, IL: Science Research Associates.

Wechsler, D. (1974). Wechsler intelligence scale for children-revised. New York: Psychological Corporation.

Yakovlev, P.I., \& Lecours, A.R. (1967). The myelogenetic cycles of regional maturation in the brain. In A. Minowski (Ed.), Regional development of the brain in early life (pp.98-114). Oxford, UK: Blackwell.

Yeudall, L.T., Fromm, D., Reddon, J.R., \& Stefanyuk, W.O. (1986). Normative data stratified by age and sex for 12 neuropsychological tests. Journal of Clinical Psychology, 42, 920-946. 
Copyright of Cognitive Neuropsychiatry is the property of Psychology Press (T\&F) and its content may not be copied or emailed to multiple sites or posted to a listserv without the copyright holder's express written permission. However, users may print, download, or email articles for individual use. 